Amelia W. Cole

University of British Columbia, Vancouver, BC, Canada

Kelsey Urgo

University of North Carolina at Chapel Hill, Chapel Hill, North Carolina, USA

\title{
Multi-method experience sampling in information behaviour research
}

\begin{abstract}
Capturing information behaviours and attitudes occurring in natural settings is a challenge. Observational methods are often intrusive or retrospective proxies, which may change behaviour or misrepresent attitudes. Technology enables novel approaches to in-situ quantitative data collection but rarely explores qualitative reflections; informing researchers on what happened, but not necessarily why. Recent work uses multi-method approaches that combine quantitative data, tracking experiences, feelings, and behaviours over time, with qualitative data to gain deeper insights into subjective experiences. This poster has two main objectives: (1) introduce experience sampling methods (ESM) to information and library scientists, and (2) show how traditional quantitative ESM measures can be extended with qualitative measures.
\end{abstract}

\section{Introduction}

The internal world of human thoughts and feelings is challenging to uncover. Current methods, such as observation, can be intrusive and proxies, such as diaries, are often retrospective.

Retrospection may be subject to cognitive biases; the feelings and attitudes experienced in the moment may not align with those reported at the time of recall (Hektner et al., 2007; Trull \& Ebner-Priemer, 2009; van Berkel et al., 2018).

\section{Experience sampling methods}

Experience sampling methods (ESM) is a longitudinal research approach that asks participants to report on their thoughts, feelings, or behaviours in context (Hektner et al., 2007). Originally designed to understand people's mental states when engaged in an activity (Csikszentmihalyi, 1997), many disciplines have since adopted ESM to document participants' daily lived experiences (Trull \& Ebner-Priemer, 2009; van Berkel et al., 2018).

In ESM, participants complete an experience sampling form (ESF) delivered in a surveylike format. The form simplifies the collection of longitudinal data by including close-ended quantitative fields (e.g., Likert scales). Participants are signalled to complete the ESF in one of four ways. (1) Random sampling captures data intermittently throughout the day. (2) Fixed sampling occurs at specific times; a useful technique when the context of the study is important (e.g., a science class). (3) Event-focused sampling commonly coincides with a self-identified triggered event, such as studying (Zirkel et al., 2015). (4) Context sampling takes advantage of sensors inside smartphones that signals participants to complete a survey based on environmental cues, such as geolocation (Intille et al., 2002; van Berkel et al., 2018).

Recent methodological advances combine quantitative and qualitative data types to gain deeper insights into lived experiences. For example, data-prompted interviews (DPI) use ESM to 
collect longitudinal quantitative data from participants followed by a personalized visual representation of the data that is subsequently used as a visual reference guide during the interview (Kwasnicka et al., 2015). For example, one study collected geographic map data during the experience sampling protocol and presented the data to participants during interviews to better understand the relationship between context and smoking behaviour (McQuoid et al., 2018).

In the field of information science, few researchers have used experience sampling methods to understand phenomena, such as information behaviour.

Two studies used a survey-like instrument to collect quantitative data with a large sample size. Rieh and colleagues conducted a study using a combination of diary methods and ESM to understand how participants $(\mathrm{N}=333)$ made credibility assessments online (Kim et al., 2009; Rieh et al., 2010). Trieu and colleagues used ESM methods to understand the relationship between interaction quality and strength of relationship ties. Participant $(\mathrm{N}=1656)$ data was collected six times a day for two weeks (Trieu et al., 2019)..

One researcher may have inadvertently used a modified ESM as part of the data collection. Zhang and colleagues collected in-situ diary data using a mix of quantitative data (e.g., queries, URLS, web page titles) and qualitative data (i.e., closed- and open-ended questions) to understand how people use search engines during creative endeavours. The researchers compiled the data into a single page layout for participant reference during the retrospective interviews (Zhang et al., 2020).

This multi-method approach adds significant value to the research on information behaviour and seeking when in-situ longitudinal data is desired. One current information research agenda well aligned to this approach is search as learning which views search as a learning process (Vakkari, 2016). A planned use of multi-method ESM explores the development of working professionals' self-efficacy when learning using search in naturalistic settings; study design employs mixed data-ESFs, visual records, and a retrospective interview.

\section{Conclusion}

Experience sampling offers information researchers a contextualized approach to data collection in longitudinal studies. This method is enhanced by creating personalised visual records that are referenced during interviews, which augments our understanding of the context of a particular phenomenon over time.

\section{Reference List:}

Csikszentmihalyi, M. (1997). Creativity: Flow and the psychology of discovery and invention. HarperPerennial.

Hektner, J., Schmidt, J., \& Csikszentmihalyi, M. (2007). Experience Sampling Method. SAGE Publications, Inc. https://doi.org/10.4135/9781412984201

Intille, S., Kukla, C., \& Ma, X. (2002). Eliciting user preferences using image-based experience sampling and reflection. Proceedings of the CHI'02 Extended Abstracts on Human Factors in Computing Systems, 2.

Kim, Y.-M., Rieh, S. Y., Yang, J. Y., \& Jean, B. S. (2009). An online activity diary method for studying credibility assessment on the Web. Proceedings of the American Society for Information Science and Technology, 46(1), 1-5. https://doi.org/10.1002/meet.2009.1450460388 
Kwasnicka, D., Dombrowski, S. U., White, M., \& Sniehotta, F. F. (2015). Data-prompted interviews: Using individual ecological data to stimulate narratives and explore meanings. Health Psychology, 34(12), 1191-1194. https://doi.org/10.1037/hea0000234

McQuoid, J., Thrul, J., \& Ling, P. (2018). A geographically explicit ecological momentary assessment (GEMA) mixed method for understanding substance use. Social Science \& Medicine, 202, 89-98. https://doi.org/10.1016/j.socscimed.2018.02.014

Rieh, S. Y., Kim, Y.-M., Yang, J. Y., \& Jean, B. S. (2010). A diary study of credibility assessment in everyday life information activities on the web: Preliminary findings. Proceedings of the American Society for Information Science and Technology, 47(1), 110. https://doi.org/10.1002/meet.14504701182

Trieu, P., Bayer, J. B., Ellison, N. B., Schoenebeck, S., \& Falk, E. (2019). Who likes to be reachable? Availability preferences, weak ties, and bridging social capital. Information, Communication \& Society, 22(8), 1096-1111. https://doi.org/10.1080/1369118X.2017.1405060

Trull, T. J., \& Ebner-Priemer, U. W. (2009). Using experience sampling methods/ecological momentary assessment (ESM/EMA) in clinical assessment and clinical research: Introduction to the special section. Psychological Assessment, 21(4), 457-462. https://doi.org/10.1037/a0017653

Vakkari, P. (2016). Searching as learning: A systematization based on literature. Journal of Information Science, 42(1), 7-18. https://doi.org/10.1177/0165551515615833

van Berkel, N., Ferreira, D., \& Kostakos, V. (2018). The Experience Sampling Method on Mobile Devices. ACM Computing Surveys, 50(6), 1-40. https://doi.org/10.1145/3123988

Zhang, Y., Capra, R., \& Li, Y. (2020). An In-situ Study of Information Needs in Design-related Creative Projects. Proceedings of the 2020 Conference on Human Information Interaction and Retrieval, 113-123. https://doi.org/10.1145/3343413.3377973

Zirkel, S., Garcia, J. A., \& Murphy, M. C. (2015). Experience-Sampling Research Methods and Their Potential for Education Research. Educational Researcher, 44(1), 7-16. JSTOR. 